Arch. Tierz., Dummerstorf 43 (2000) 6, 633-647

Research Institute for the Biology of Farm Animals (FBN), Dummerstorf, Germany

ELKE SAGGAU, MANFRED BEYER, MONIKA KLEIN, RUTHILD SCHADEREIT, MICHAEL DERNO, WERNER JENTSCH and HELMUT SCHOLZE

\title{
Effects of dietary protein quality on energy metabolism and thyroid hormone status in growing pigs
}

Dedicated to Prof. Dr. habil. agr. H. Hagemeister on the occasion of his $65^{\text {th }}$ birthday

\section{Summary}

To estimate long-term effects of dietary protein quality on energy metabolism and thyroid hormone status in growing pigs two experiments were carried out, each using 6 growing German Landrace barrows (40 to $90 \mathrm{~kg}$ body weight $(\mathrm{BW})$ ) per treatment group, which were fed semisynthetic isoenergetic diets based on either casein or soy protein isolate at $1875 \mathrm{~kJ} \mathrm{ME} /\left(\mathrm{kg} \mathrm{BW}^{0.62} \mathrm{x} \mathrm{d}\right)$. Casein was tested with $(\mathrm{CAS}+)$ amino acid (AA) supplementation (methionine + cystine, threonine, tryptophane) and soy protein isolate was tested without (SPI-) AA supplementation at the recommended protein supply of $100 \%$ (normal protein level (NP)) and at a protein supply of $50 \%$ of NP. During experiments pigs were housed individually in metabolic cages at $23 \pm 1^{\circ} \mathrm{C}$. At both protein supply levels, SPI- in comparison to CAS + caused a lower protein energy retention (PER), which was compensated mainly by an increased fat energy retention (FER). The reduction of the protein supply to $50 \%$ caused a lower PER by 30 to $50 \%$ in both dietary qualities, which was compensated by a significantly higher FER. However, the heat production (HP) was neither affected by the protein quality nor by the quantity, and resulted in nearly similar values of $60 \%$ of $\mathrm{ME}$ intake. The thyroid hormone concentrations were dependent primarily on the amount of protein supply, and after decrease of supply to $50 \%$ secondly on the dietary protein quality. The increased thyroid hormone concentrations at the $50 \%$ protein level were in euthyroid range of pigs and obviously not associated with HP.

Key Words: protein quality, soy protein isolate, casein, thyroid hormones, energetic efficiency, pigs

\section{Zusammenfassung}

Titel der Arbeit: Effekte der Nahrungsproteinqualităt auf den Energieumsatz und den Schilddrüsenhormonstatus wachsender Schweine

Um Langzeiteffekte von Nahrungsproteinqualităten auf den Energieumsatz und den Schilddrüsenhormonstatus wachsender Schweine zu bestimmen, wurden 2 Versuche mit jeweils 6 wachsenden Börgen der Deutschen Landrasse ( 40 bis $90 \mathrm{~kg} \mathrm{LM}$ ) je Versuchsgruppe durchgefuhrt. Die Tiere wurden mit semisynthetischen, isoenergetischen Dialten $\left(1875 \mathrm{~kJ} \mathrm{ME} /\left(\mathrm{kg} \mathrm{BW}{ }^{0.62} \times \mathrm{d}\right)\right.$ ) gefuttert, die Casein oder Sojaproteinisolat als alleinige Proteinquelle enthielten. Casein (CAS+) wurde mit Aminosăurenergänzung (Methionin + Cystein, Threonin, Tryptophan) und Sojaproteinisolat (SPI-) ohne AA-Ergänzung auf einer $100 \%$ igen Proteinversorgungsstufe (normal protein level, NP) und einer 50 \%igen (low protein level (LP)) getestet. Wăhrend der Versuche wurden die Tiere einzeln in Stoffwechselkäfigen bei $23 \pm 1^{\circ} \mathrm{C}$ gehalten. Auf beiden Proteinversorgungsstufen fulhrte die Fütterung von SPI- im Vergleich zu CAS+ zu einer signifikant niedrigeren Proteinenergieretention (PER), die uberwiegend durch eine høhere Fettenergieretention (FER) kompensiert wurde. Die Senkung der Proteinversorgung auf $50 \%$ hatte fur beide Proteinqualităten eine 30 bis $50 \%$ niedrigere PER zur Folge, welches durch eine höhere FER kompensiert wurde. Die Wărmeproduktion (WP) wurde weder durch die Proteinqualităt noch durch die -quantităt beeinflusst und resultierte in nahezu gleichen Werten von $60 \%$ der Einnahme an ME. Die Schilddrusenhormonkonzentrationen waren in erster Linie von der Proteinversorgungsstufe abhăngig und erst nach Senkung der Proteingabe auf 50 \% von der Qualităt. Die erhöhten Schilddrüsenhormonwerte auf LP lagen im euthyroiden Bereich und waren mit der WP offensichtlich nicht verbunden.

Schlusselworter: Proteinqualităt, Sojaproteinisolat, Casein, Schilddrulsenhormone, Gesamtenergieverwertung, Schweine 
Introduction

Long-term feeding of diets, which meet the energy requirements for maintenance and growth, but not the requirement for essential amino acids (AA), decreases growth rate and protein deposition in growing animals. The excess of dietary energy, which cannot be deposited as protein can either be used for fat deposition (KEAGY et al., 1987) or dissipated as heat (TULP et al., 1979; GURR et al., 1980). Although several studies were carried out to measure energy balance under these conditions, only a few studies have attempted to investigate the regulatory factors of thermogenesis. It is known that thyroid hormones may play a role in mediating the thermogenic response to low protein diets.

Less research has been done in examining the effect of feeding dietary proteins with a low biological value on energy metabolism of growing animals. From previous data one cannot conclude unambiguously whether specific AA patterns are responsible for differences in efficiency of the utilization of metabolizable energy (ME). It is observed that long-term feeding of plant protein (e.g. soy protein, wheat gluten) with somewhat lower biological value, in comparison to high quality animal proteins (casein, egg protein) induced higher serum thyroid hormone levels, particularly higher total thyroxine $\left(\mathrm{T}_{4}\right)$ levels, in monogastric animals (CREE and SCHALCH, 1985; FORSYTHE, 1986; BARTH et al., 1988; SCHOLZ-AHRENS et al., 1990; POTTER et al., 1996). The question arises whether a higher $\mathrm{T}_{4}$ level in AA deficient fed animals represents a true change in biological activity and does play a role in dissipating excess dietary energy.

The objective of the present study was to investigate the influence of dietary protein quality (AA pattern) on energy metabolism and thyroid hormone status, as well as to explore whether differences in energetic efficiency are associated with changes in circulating thyroid hormone levels.

\section{Materials and methods}

\section{Animals and diets}

Two separate experiments were carried out each with six castrated male pigs of the German Landrace, weighing between 30 and $90 \mathrm{~kg}$. At the initial body weight (BW) of $30 \mathrm{~kg}$ animals were equipped with permanent vein catheters for stress-free blood sampling. During the experiments the pigs were housed individually in metabolic cages. To meet the thermic demands of the animals the environmental temperature at the start of the trials was kept at $25^{\circ} \mathrm{C}$. With increasing body weight the temperature was decreased up to $22^{\circ} \mathrm{C}$. The relative humidity was $60-70 \%$. They were fed twice daily with semisynthetic, isoenergetic diets, which provided 2.5 times the maintenance requirement for metabolizable energy (1875 $\mathrm{kJ} \mathrm{ME} /\left(\mathrm{kg} \mathrm{BW}^{0.62} \mathrm{x}\right.$ d). This corresponded to a daily feed intake of approximately $110 \mathrm{~g} \mathrm{DM} / \mathrm{kg} \mathrm{BW}^{0.62}$. Water was offered to ad libitum intake. To enable a comparison with human nutrition, nutrient composition of the diets was similar to the composition of human diets in western industrial countries (protein, 8-18\%; starch, 32-42\%; sucrose, 20\%; fat, $15 \%$; cellulose, 7\%; mineral and vitamin $\mathrm{mix}, 8 \%$, wt/wt). Protein and starch content were 
altered, depending on age and level of protein supply. Casein was supplemented with limiting essential AA (Met + Cys, Thr, Trp) to the level recommended by the GfEH (1987). Correspondingly, CAS+ was supplemented with $1,15 \mathrm{~g} \mathrm{Met}, 0.58 \mathrm{~g}$ Thr and $0.46 \mathrm{~g}$ Trp per $16 \mathrm{~g} \mathrm{~N}$. Soy protein isolate was tested without (SPI-) supplementation. In the SPI- treatment the AA Lys, Met + Cys, Thr and Trp, provided 74, 58, 75 and $73 \%$ of the recommended level, respectively. For pre-calculation of $\mathrm{ME}$ values in diets $(\mathrm{kJ} / \mathrm{kg} \mathrm{DM})$, the equation of HOFFMANN et al. (1993) was used. Effects of the dietary protein quality were compared at normal protein level (NP, experiment 1) and at low protein level (LP, $50 \%$ of NP, experiment 2).

\section{Treatments and experimental procedures}

In both experiments, trial periods were performed to the same pattern: 10 days of preperiod and 8 days of main period with determinations of energy and protein balance ( $\mathrm{CN}$ balance), including 4 days of measurements of gaseous exchange in the respiration chamber. During the main period pigs were kept in metabolic cages allowing daily collection of urine and faeces separately. The body weight of the animals was recorded weekly and health status was monitored by daily measuring of rectal temperature. Each experiment was performed as a cross-over trail, i. e. after the $2^{\text {nd }}$ period the dietary proteins CAS + and SPI- were replaced by one another. Pigs were given 21 days to adapt to the new experimental conditions and diets. Measurements of $\mathrm{CN}$ balance were done in all periods and determination of thyroid hormones in the $2^{\text {nd }}$ and $4^{\text {th }}$ period. For thyroid hormone determination blood samples $(10 \mathrm{~mL} /$ day $)$ were taken during the main period prior to the morning feeding. Each sample was allowed to clot for 12 hours, followed by centrifugation with $3000 \mathrm{xg}$ at $4^{\circ} \mathrm{C}$. Aliquots of sera were taken and stored at $-20^{\circ} \mathrm{C}$ until assayed for thyroid hormones.

\section{Analytical methods}

The energy content of feed, freeze-dried faeces and urine was determined with an adiabatic bomb calorimeter (C400; JANKE \& KUNKEL GmbH, Staufen, Germany). The amino acids were analyzed by ion-exchange chromatography using an amino acid analyser BIOCHROM 20 (PHARMACIA-BIOTECH EUROPE GmbH, Freiburg, Germany). For all other analyses of feed, faeces and urine conventional methods of the Verband Deutscher landwirtschaftlicher Untersuchungs- und Forschungsanstalten (VDLUFA, 1988) were used.

The energy balance was measured by indirect calorimetry based on the carbon and nitrogen balances. Energy retention was calculated by using the factors given by BROWER (1965) and HOFFMANN and SCHIEMANN (1980). Heat production was calculated as difference between ME intake and energy retention.

Thyroid hormones, total thyroxine $\left(\mathrm{T}_{4}\right)$, total triiodothyronine $\left(\mathrm{T}_{3}\right)$ and the free forms, $\mathrm{fT}_{4}$ and $\mathrm{fT}_{3}$, were determined by radioimmunoassays (RIA-COAT, BYK-SANTEC DIAGNOSTICA GmbH \& Co.KG, Dietzenbach, Germany).

\section{Statistical analysis}

Effects of protein sources within periods were evaluated by one-way ANOVA using 
SPSS (Statistical Package for the Social Science, Version 7.5, Chicago, 1997). All the results presented in tables are mean values with standard deviations. Differences were considered to be significant at $\mathrm{p}<0.05$.

\section{Results}

Nutrients and energy of both diets were highly digestible (approximately $90 \%$, data not shown). The apparent $\mathrm{N}$ digestibility was influenced by both the protein quality and quantity and ranged between 89.9 and 93.5 at NP and between 82.9 and $87.1 \%$ at the LP level, always with the lower values in the SPI- groups.

With the exception of the $2^{\text {nd }}$ period of NP the differences in the intake of metabolizable energy between CAS+ and SPI- within periods were not significantly different (Table 1). Generally the intake of ME in SPI- fed pigs was slightly lower. Within the periods of NP the intake of protein per $\mathrm{kg} \mathrm{BW}^{0.62}$ was not significantly different. At LP the intake of protein per $\mathrm{kg} \mathrm{BW}^{0.62}$ within the periods was slightly but significantly lower for SPI- than for CAS+.

Both, protein quality and quantity, affected the daily weight gain of pigs (Table 1). At NP the values for CAS+ fed pigs in the $1^{\text {st }}$ and $2^{\text {nd }}$ period were 504 and $567 \mathrm{~g}$, respectively, which was in both periods approximately $90 \mathrm{~g}$ higher than the daily gain of the SPI- pigs. After cross-over feeding, the difference between CAS+ and SPIincreased to $130 \mathrm{~g} / \mathrm{d}$ in the $3^{\text {rd }}$ period; in the $4^{\text {th }}$ period the difference was $86 \mathrm{~g} / \mathrm{d}$. In all periods of NP the differences were statistically significant. Reduction of the protein content to $50 \%$ (LP) generally resulted in a reduced growth performance in both dietary groups. The daily gain of CAS+ fed pigs amounted from $302 \mathrm{~g}$ in the $1^{\text {st }}$ to 494 $\mathrm{g}$ in the $4^{\text {th }}$ period, and of SPI- fed pigs from 262 to $406 \mathrm{~g}$, respectively. With the exception of the $3^{\text {rd }}$ period, after cross-over feeding, differences in daily gain between CAS+ and SPI- groups remained significant. The final BW within the same time period was approximately $90 \mathrm{~kg}$ at the NP and approximately $60 \mathrm{~kg}$ at the LP feeding level.

At almost the same ME intake levels, protein energy retention (PER) was markedly affected by protein quality and quantity. In the $1^{\text {st }}$ period of NP, feeding of SPIresulted in a significantly lower PER than feeding of CAS+ $(205 \mathrm{vs.} 279 \mathrm{~kJ})$. The values of PER in the $2^{\text {nd }}$ period decreased for CAS+ by $27 \%$ and for SPI- by $18 \%$ compared to the $1^{\text {st }}$ period. However, the difference between both protein sources was significant. After cross-over feeding, the values of PER for SPI- remained constant but the value for CAS+ increased to $221 \mathrm{~kJ}$. In the $4^{\text {th }}$ period the values of PER decreased further for both protein sources, however the differences between CAS+ and SPIremained significant.

As the PER during growth decreased, the fat energy retention (FER) increased for $\mathrm{CAS}+$ from $394 \mathrm{~kJ}$ in the $1^{\text {st }}$ period to $715 \mathrm{~kJ}$ in the $4^{\text {th }}$ period, and for SPI- from 447 to $642 \mathrm{~kJ}$, respectively. Also, the total energy retention (ER) increased from $673 \mathrm{~kJ}$ for CAS+ and $651 \mathrm{~kJ}$ for SPI- in the $1^{\text {st }}$ period to 882 and $785 \mathrm{~kJ}$ in the $4^{\text {th }}$ period, respectively. Correspondingly, heat production (HP) decreased for both treatments. Although in all periods of NP feeding of SPI- as compared to CAS+ resulted in a significantly lower PER, no significant differences in values of FER (exception of $4^{\text {th }}$ 
Table 1

Energy and $\mathrm{N}$ balance data of growing pigs fed different dietary protein qualities and quantities (Means and standard deviations). (Energie- und $\mathrm{N}$-Bilanzdaten wachsender Schweine bei Fütterung unterschiedlicher Nahrungsproteinqualităten und -quantităten (Mittelwerte und Standardabweichungen))

\begin{tabular}{|c|c|c|c|c|c|c|c|c|c|}
\hline Period & $\begin{array}{l}\text { Protein } \\
\text { source }\end{array}$ & $\begin{array}{c}\mathrm{n} \\
\text { animals }\end{array}$ & $\begin{array}{l}\text { Mean } \\
\mathrm{BW}^{\mathrm{t}} \\
(\mathrm{kg})\end{array}$ & $\begin{array}{l}\text { Weight } \\
\text { gain } \\
\text { (g/d) }\end{array}$ & $\begin{array}{c}\text { ME intake } \\
\mathrm{kJ} /(\mathrm{kg} \\
\left.\mathrm{BW}^{0.62} \times \mathrm{d}\right)\end{array}$ & $\begin{array}{l}\text { Protein intake } \\
\mathrm{g} /(\mathrm{kg} \\
\left.\mathrm{BW}^{0.62} \times \mathrm{d}\right)\end{array}$ & $\begin{array}{c}\text { Energy } \\
\text { retention (ER) }\end{array}$ & $\begin{array}{cc}\text { Protein } & \text { Fat } \\
\text { ER } & \text { ER } \\
\mathrm{kJ} /\left(\mathrm{kg} \mathrm{BW}^{0.62} \times \mathrm{d}\right)\end{array}$ & $\begin{array}{c}\text { Heat } \\
\text { production }\end{array}$ \\
\hline
\end{tabular}

Experiment 1: Normal protein level, NP (Protein supply: 100\%)

$\begin{array}{cllll}\text { CAS+ } & 5^{*} & 88.3 \pm 3.2 & 763 \pm 21^{2} & 1919 \pm 26^{\circ} \\ \text { SPI- } & 5^{*} & 83.3 \pm 4.1 & 677 \pm 30^{\mathrm{b}} & 1889 \pm 46^{2}\end{array}$

Experiment 2: Low protein level, LP (Protein supply: $50 \%$ of NP)

\begin{tabular}{|c|c|c|c|c|c|c|c|c|c|c|}
\hline 1 & $\begin{array}{l}\text { CAS+ } \\
\text { SPI- }\end{array}$ & $\begin{array}{l}6 \\
6\end{array}$ & $\begin{array}{l}32.2 \pm 1.5 \\
32.1 \pm 0.8\end{array}$ & $\begin{array}{l}302 \pm 28^{2} \\
262 \pm 31^{b}\end{array}$ & $\begin{array}{l}1924 \pm 60^{2} \\
1917 \pm 49^{2}\end{array}$ & $\begin{array}{l}9.1 \pm 0.1^{a} \\
9.6 \pm 0.1^{b}\end{array}$ & $\begin{array}{l}751 \pm 73^{\mathrm{a}} \\
740 \pm 73^{\mathrm{a}}\end{array}$ & $\begin{array}{l}144 \pm 7^{a} \\
89 \pm 12^{b}\end{array}$ & $\begin{array}{l}607 \pm 69^{a} \\
651 \pm 68^{a}\end{array}$ & $\begin{array}{l}1173 \pm 48^{2} \\
1178 \pm 68^{2}\end{array}$ \\
\hline 2 & $\begin{array}{l}\text { CAS+ } \\
\text { SPI- }\end{array}$ & $\begin{array}{l}6 \\
6\end{array}$ & $\begin{array}{l}38.7 \pm 3.1 \\
37.7 \pm 0.8\end{array}$ & $\begin{array}{l}343 \pm 28^{a} \\
298 \pm 48^{b}\end{array}$ & $\begin{array}{l}1921 \pm 51^{2} \\
1875 \pm 43^{2}\end{array}$ & $\begin{array}{l}8.9 \pm 0.1^{\mathrm{a}} \\
9.6 \pm 0.2^{\mathrm{b}}\end{array}$ & $\begin{array}{l}783 \pm 88^{a} \\
724 \pm 39^{a}\end{array}$ & $\begin{array}{c}148 \pm 9^{a} \\
96 \pm 10^{b}\end{array}$ & $\begin{array}{l}635 \pm 81^{a} \\
629 \pm 35^{2}\end{array}$ & $\begin{array}{l}1137 \pm 42^{a} \\
1150 \pm 57^{a}\end{array}$ \\
\hline 3 & $\begin{array}{l}\text { CAS+ } \\
\text { SPI- }\end{array}$ & $\begin{array}{l}6 \\
4^{* *}\end{array}$ & $\begin{array}{l}49.3 \pm 1.3 \\
51.1 \pm 1.6\end{array}$ & $\begin{array}{l}358 \pm 33^{a} \\
337 \pm 17^{2}\end{array}$ & $\begin{array}{l}1831 \pm 35^{\mathrm{a}} \\
1812 \pm 9^{\mathrm{a}}\end{array}$ & $\begin{array}{l}8.0 \pm 0.1^{a} \\
8.4 \pm 0.2^{b}\end{array}$ & $\begin{array}{l}699 \pm 44^{2} \\
687 \pm 15^{2}\end{array}$ & $\begin{aligned} 114 & \pm 5^{a} \\
83 & \pm 5^{b}\end{aligned}$ & $\begin{array}{l}585 \pm 40^{2} \\
604 \pm 12^{a}\end{array}$ & $\begin{array}{l}1132 \pm 57^{\mathrm{a}} \\
1125 \pm 17^{\mathrm{a}}\end{array}$ \\
\hline 4 & $\begin{array}{l}\text { CAS+ } \\
\text { SPI- }\end{array}$ & $\begin{array}{l}6 \\
4^{*=}\end{array}$ & $\begin{array}{l}57.2 \pm 2.4 \\
58.3 \pm 1.7 \\
\end{array}$ & $\begin{array}{l}494 \pm 54^{a} \\
406 \pm 41^{b}\end{array}$ & $\begin{array}{l}1816 \pm 61^{2} \\
1828 \pm 40^{2}\end{array}$ & $\begin{array}{l}7.9 \pm 0.1^{a} \\
8.4 \pm 0.2^{b}\end{array}$ & $\begin{array}{l}742 \pm 53^{a} \\
720 \pm 55^{a}\end{array}$ & $\begin{array}{l}119 \pm 2^{\mathrm{a}} \\
82 \pm 7^{\mathrm{b}}\end{array}$ & $\begin{array}{l}623 \pm 52^{2} \\
638 \pm 48^{3}\end{array}$ & $\begin{array}{l}1074 \pm 57^{\mathrm{a}} \\
1108 \pm 33^{\mathrm{a}}\end{array}$ \\
\hline
\end{tabular}

Protein sources were cascin with amino acid supplementation (CAS+) and soy protein isolate without amino acid supplementation (SPI-). Experiments werc cross over trials,

i. e. after two periods dietary proteins CAS+ and SPI- were replaced by each other.' $M$ Mean body weight $(\mathrm{kg})$ of the group at middle of the respective period.

-

$13.5 \pm 0.2^{2}$

$882 \pm 46^{\mathrm{a}}$
$785 \pm 37^{\mathrm{b}}$

$143 \pm 14$

$642 \pm 38$

$104 \pm 51$

$\begin{array}{lll}279 \pm 25^{\mathrm{a}} & 394 \pm 86^{\mathrm{a}} & 1217 \pm 67^{\mathrm{a}} \\ 205 \pm 9^{\mathrm{b}} & 447 \pm 50^{\mathrm{a}} & 1186 \pm 58^{\mathrm{a}} \\ & & \\ 205 \pm 6^{\mathrm{a}} & 542 \pm 43^{\mathrm{a}} & 1157 \pm 65^{\mathrm{a}} \\ 168 \pm 8^{\mathrm{b}} & 541 \pm 74^{\mathrm{a}} & 1125 \pm 59^{\mathrm{a}} \\ & & \\ 221 \pm 16^{\mathrm{a}} & 600 \pm 52^{\mathrm{a}} & 1109 \pm 61^{\mathrm{a}} \\ 167 \pm 7^{\mathrm{b}} & 612 \pm 44^{\mathrm{a}} & 1119 \pm 72^{\mathrm{a}} \\ 168 \pm 5^{\mathrm{a}} & 715 \pm 48^{\mathrm{a}} & 1037 \pm 39^{\mathrm{a}} \\ 143 \pm 14^{\mathrm{b}} & 642 \pm 38^{\mathrm{b}} & 1104 \pm 51^{\mathrm{a}}\end{array}$


period), as well as of HP, between CAS+ and SPI- were determined.

In Table 2 the partition of ME into PER and FER as well as into ER (total efficiency of utilization of ME) and HP in \% are calculated. With exception of the $4^{\text {th }}$ period, feeding of SPI- in comparison to CAS+ resulted in a significantly lower proportion of $\mathrm{ME}$ in PER; values of FER/ME and ER/ME as correspondingly HP/ME were not significantly different but always on an average slightly higher for SPI-. There was a decrease of PER/ME and an increase of FER/ME for both dietary groups during growth of the animals. In the periods 1 to 3, CAS + fed pigs showed significantly higher values of PER/ER than SPI-fed pigs. During growth, as the values of PER/ER decreased the values of FER/ER increased. Additionally, the differences between CAS+ and SPI- diminished with increasing $\mathrm{BW}$, however in the $4^{\text {th }}$ period no significant differences were observed.

The reduction of protein supply to $50 \%$ (LP) resulted in a similar HP than at NP (Table 1). At LP markedly lower PER (50 to $60 \%$ ) and higher FER (30 to $40 \%$ ) than at NP in both dietary groups were observed. During growth PER decreased slightly for CAS+ as well as for SPI-, however the differences between both protein sources remained significant. In all periods of LP for FER and HP no significant differences between CAS+ and SPI- were observed.

The proportions of HP in ME (Table 2) between CAS+ and SPI- were not significantly different in all periods of LP; both dietary groups showed an average value of $60 \%$. Feeding of SPI- resulted in a significantly lower proportion of PER in ME than feeding CAS+, FER/ME was not affected by the dietary treatment. There was a decrease of $\mathrm{PER} / \mathrm{ME}$ and an increase of FER/ME for both dietary groups during growth of pigs. In all periods of LP CAS+ resulted in a significantly higher ratio of PER/ER and in a lower ratio of FER/ER than SPI-. During growth the values of PER/ER decreased for CAS+ from 19.2 to $16.0 \%$; for SPI- the values remained constant at about $12 \%$.

\section{Thyroid hormones}

Concentrations of thyroid hormones in the serum measured at both protein levels in the $2^{\text {nd }}$ and $4^{\text {th }}$ fattening period are summarized in Table 3 . Thyroid hormone levels were constant within periods, which allowed to calculate an average for each variable $\left(\mathrm{T}_{4}, \mathrm{~T}_{3}, \mathrm{fT}_{4}, \mathrm{fT}_{3}\right)$ for each period.

At NP, serum concentrations of thyroid hormones were not affected by the dietary protein quality. The ratio of $\mathrm{T}_{3} / \mathrm{T}_{4}$ was not affected by the dietary protein quality or by the period. In the $4^{\text {th }}$ period similar thyroid hormone concentrations were determined as in the $2^{\text {nd }}$ period.

At LP, serum concentrations of thyroid hormones were affected by the dietary protein quality. In the $2^{\text {nd }}$ period for SPI- in comparison to CAS+ not significantly but slightly higher concentrations of $\mathrm{T}_{4}(56.28$ vs. $50.84 \mathrm{nmol} / \mathrm{L})$ and significantly higher concentrations of $\mathrm{fT}_{4}(16.15$ vs. $11.91 \mathrm{pmol} / \mathrm{L})$ were observed. In contrast, significantly lower values of $\mathrm{T}_{3}(1.57 \mathrm{vs} .2 .32 \mathrm{nmol} / \mathrm{L})$ and $\mathrm{fT}_{3}(0.31 \mathrm{vs} .0 .75 \mathrm{pmol} / \mathrm{L})$ were determined compared to the CAS + . Similar observations were made in the $4^{\text {th }}$ period.

The reduction of protein supply to $50 \%(\mathrm{LP})$ caused a markedly higher $\mathrm{T}_{4}$ concent- 
Table 2

Partition of metabolizable energy (ME) into protein energy retention (PER), fat energy retention (FER), total energy retention (ER) and heat production (HP) as well as ratios of PER to ER and FER to ER in growing pigs fed different protein qualities and quantities (Means and standard deviations). (Aufteilung der umsetzbaren Energie (ME) in Proteinenergieretention (PER), Fettenergieretention (FER), Gesamtenergieretention (ER) und Wärmeproduktion (HP) sowie die Verhăltnisse von PER zu ER und FER zu ER in wachsenden Schweinen bei der Fütterung unterschiedlicher Nahrungsproteinqualitäten und -quantitäten (Mittelwerte und Standardabweichungen))

\begin{tabular}{|c|c|c|c|c|c|c|c|c|}
\hline Period & $\begin{array}{l}\text { Protein } \\
\text { source }\end{array}$ & $\begin{array}{l}\mathrm{n} \\
\text { animals }\end{array}$ & $\begin{array}{c}\text { PER/ME } \\
(\%)\end{array}$ & $\begin{array}{c}\text { FER/ME } \\
(\%)\end{array}$ & $\begin{array}{c}\text { ER/ME } \\
(\%)\end{array}$ & $\begin{array}{c}\mathrm{HP} / \mathrm{ME} \\
(\%)\end{array}$ & $\begin{array}{c}\text { PER/ER } \\
(\%)\end{array}$ & $\begin{array}{c}\text { FER/ER } \\
(\%)\end{array}$ \\
\hline \multicolumn{9}{|c|}{ xperiment 1: Normal protein level, NP (Protein supply 100\%) } \\
\hline 1 & $\begin{array}{l}\text { CAS+ } \\
\text { SPI- }\end{array}$ & $\begin{array}{l}6 \\
6\end{array}$ & $\begin{array}{l}14.7 \pm 1.1^{a} \\
11.1 \pm 0.3^{b}\end{array}$ & $\begin{array}{l}20.8 \pm 4.2^{2} \\
24.3 \pm 2.6^{2}\end{array}$ & $\begin{array}{l}35.6 \pm 4.3^{a} \\
35.4 \pm 2.9^{a}\end{array}$ & $\begin{array}{l}64.4 \pm 4.3^{a} \\
64.6 \pm 2.9^{a}\end{array}$ & $\begin{array}{l}41.8 \pm 5.1^{\mathrm{a}} \\
31.5 \pm 1.9^{\mathrm{b}}\end{array}$ & $\begin{array}{l}58.2 \pm 5.1^{a} \\
68.5 \pm 2.0^{b}\end{array}$ \\
\hline 2 & $\begin{array}{l}\text { CAS+ } \\
\text { SPI- }\end{array}$ & $\begin{array}{l}6 \\
6\end{array}$ & $\begin{aligned} 10.8 & \pm 0.4^{a} \\
9.1 & \pm 0.5^{b}\end{aligned}$ & $\begin{array}{l}28.5 \pm 2.6^{a} \\
29.5 \pm 3.6^{2}\end{array}$ & $\begin{array}{l}39.3 \pm 2.7^{2} \\
38.6 \pm 3.7^{4}\end{array}$ & $\begin{array}{l}60.7 \pm 2.7^{a} \\
61.4 \pm 3.7^{a}\end{array}$ & $\begin{array}{l}27.6 \pm 1.6^{a} \\
23.8 \pm 2.1^{b}\end{array}$ & $\begin{array}{l}72.4 \pm 1.6^{a} \\
76.2 \pm 2.1^{b}\end{array}$ \\
\hline 3 & $\begin{array}{l}\text { CAS+ } \\
\text { SPI- }\end{array}$ & $\begin{array}{l}6 \\
6\end{array}$ & $\begin{array}{r}11.5 \pm 0.8^{a} \\
8.8 \pm 0.5^{b}\end{array}$ & $\begin{array}{l}31.1 \pm 3.0^{a} \\
32.3 \pm 2.6^{a}\end{array}$ & $\begin{array}{l}42.5 \pm 2.6^{a} \\
41.1 \pm 3.0^{2}\end{array}$ & $\begin{array}{l}57.5 \pm 2.6^{2} \\
58.9 \pm 3.0^{a}\end{array}$ & $\begin{array}{l}27.0 \pm 2.9^{a} \\
21.5 \pm 0.8^{b}\end{array}$ & $\begin{array}{l}73.0 \pm 3.0^{a} \\
78.5 \pm 0.8^{b}\end{array}$ \\
\hline $\begin{array}{c}4 \\
\text { perimen }\end{array}$ & $\begin{array}{c}\text { CAS+ } \\
\text { SPI- } \\
\text { Low prote }\end{array}$ & $\begin{array}{c}5^{*} \\
5^{*} \\
\text { vel, LP (P }\end{array}$ & $\begin{array}{c}8.2 \pm 1.3^{\mathrm{a}} \\
7.1 \pm 1.1^{\mathrm{a}} \\
\text { supply: } 50 \%\end{array}$ & $\begin{array}{l}37.4 \pm 2.1^{a} \\
34.8 \pm 2.8^{a}\end{array}$ & $\begin{array}{l}45.6 \pm 2.1^{\mathrm{a}} \\
41.1 \pm 2.1^{\mathrm{b}}\end{array}$ & $\begin{array}{l}54.4 \pm 2.1^{\mathrm{a}} \\
58.1 \pm 2.1^{\mathrm{b}}\end{array}$ & $\begin{array}{l}18.0 \pm 2.8^{2} \\
17.1 \pm 3.1^{2}\end{array}$ & $\begin{array}{l}82.0 \pm 2.8^{\mathrm{a}} \\
82.9 \pm 3.1^{\mathrm{a}}\end{array}$ \\
\hline 1 & $\begin{array}{l}\text { CAS+ } \\
\text { SPI- }\end{array}$ & $\begin{array}{l}6 \\
6\end{array}$ & $\begin{array}{l}7.5 \pm 0.2^{\mathrm{a}} \\
4.6 \pm 0.6^{\mathrm{b}}\end{array}$ & $\begin{array}{l}31.5 \pm 3.0^{\mathrm{a}} \\
34.0 \pm 3.4^{\mathrm{a}}\end{array}$ & $\begin{array}{l}39.0 \pm 3.0^{2} \\
38.6 \pm 3.5^{2}\end{array}$ & $\begin{array}{l}61.0 \pm 3.0^{\mathrm{a}} \\
61.4 \pm 3.5^{\mathrm{a}}\end{array}$ & $\begin{array}{l}19.2 \pm 0.9^{\mathrm{a}} \\
12.0 \pm 1.6^{\mathrm{b}}\end{array}$ & $\begin{array}{l}80.8 \pm 1.6^{\mathrm{a}} \\
88.0 \pm 1.6^{b}\end{array}$ \\
\hline 2 & $\begin{array}{l}\text { CAS+ } \\
\text { SPI- }\end{array}$ & $\begin{array}{l}6 \\
6\end{array}$ & $\begin{array}{l}7.7 \pm 0.3^{a} \\
5.1 \pm 0.5^{b}\end{array}$ & $\begin{array}{l}33.0 \pm 3.4^{a} \\
33.6 \pm 0.7^{a}\end{array}$ & $\begin{array}{l}40.7 \pm 3.5^{\mathrm{a}} \\
38.7 \pm 2.3^{\mathrm{a}}\end{array}$ & $\begin{array}{l}59.3 \pm 3.5^{\mathrm{a}} \\
61.3 \pm 2.3^{\mathrm{a}}\end{array}$ & $\begin{array}{r}19.2 \pm 1.8^{\mathrm{a}} \\
13.2 \pm 1.2^{\mathrm{b}}\end{array}$ & $\begin{array}{l}80.9 \pm 1.3^{\mathrm{a}} \\
86.7 \pm 1.2^{\mathrm{b}}\end{array}$ \\
\hline 3 & $\begin{array}{l}\text { CAS+ } \\
\text { SPI- }\end{array}$ & $\begin{array}{c}6 \\
4^{* *}\end{array}$ & $\begin{array}{l}6.2 \pm 0.2^{a} \\
4.6 \pm 0.3^{b}\end{array}$ & $\begin{array}{l}32.0 \pm 2.3^{\mathrm{a}} \\
33.4 \pm 0.7^{\mathrm{a}}\end{array}$ & $\begin{array}{l}38.2 \pm 2.5^{a} \\
37.9 \pm 0.9^{a}\end{array}$ & $\begin{array}{l}61.8 \pm 2.5^{a} \\
62.1 \pm 0.9^{a}\end{array}$ & $\begin{array}{l}16.2 \pm 0.4^{a} \\
12.0 \pm 0.5^{b}\end{array}$ & $\begin{array}{l}83.7 \pm 0.7^{a} \\
88.0=0.5^{b}\end{array}$ \\
\hline 4 & $\begin{array}{l}\text { CAS+ } \\
\text { SPI- }\end{array}$ & $\begin{array}{c}6 \\
4^{* * *} \\
\end{array}$ & $\begin{array}{l}6.5 \pm 0.2^{a} \\
4.5 \pm 0.3^{b} \\
\end{array}$ & $\begin{array}{l}34.3 \pm 2.6^{\mathrm{a}} \\
34.9 \pm 2.0^{\mathrm{a}} \\
\end{array}$ & $\begin{array}{l}40.7 \pm 2.6^{a} \\
39.3 \pm 2.4^{a} \\
\end{array}$ & $\begin{array}{l}59.2 \pm 2.6^{a} \\
60.6 \pm 2.4^{a}\end{array}$ & $\begin{array}{l}16.0 \pm 0.9^{\mathrm{a}} \\
11.4 \pm 0.3^{b}\end{array}$ & $\begin{array}{l}84.0 \pm 1.2^{a} \\
88.6 \pm 0.3^{b} \\
\end{array}$ \\
\hline
\end{tabular}

Protein sources were casein with amino acid supplementation (CAS+) and soy protein isolate without amino acid supplementation (SPI-). Experiments were cross over

trials, i. e. after two periods dietary proteins CAS+ and SPI- were replaced by each other.

${ }^{2 b}$ Means with different superscripts within a period and within one column are significantly different $(p<0.05)$

- One animal was taken out of experiments duc to health problems; ** Two animals were taken out of experiments due to health problems. 
Table 3

Concentration of thyroid hormones $\left(\mathrm{T}_{4}, \mathrm{~T}_{3}, \mathrm{fT}_{4} \text { and } \mathrm{fT}_{3}\right)^{1}$ in the serum of growing pigs fed different dietary protein qualities and quantities (Means and standard deviations). (Schilddrusenhormonkonzentration $\left(\mathrm{T}_{4}, \mathrm{~T}_{3}\right.$, $\mathrm{fT}_{4}$ and $\left.\mathrm{fT}_{3}\right)^{1}$ im Serum wachsender Schweine bei Futterung unterschiedlicher Nahrungsproteinqualităten und -quantităten (Mittelwerte und Standardabweichungen))

\begin{tabular}{cccccccc}
\hline Period & Protein & $\mathrm{n}$ & Total $\mathrm{T}_{4}$ & Free $\mathrm{T}_{4}$ & Total $\mathrm{T}_{3}$ & $\mathrm{Free}_{3}$ & $\mathrm{~T}_{3} / \mathrm{T}_{4}$ \\
& source & pigs & $\left(\mathrm{T}_{4}\right)$ & $\left(\mathrm{fT}_{4}\right)$ & $\left(\mathrm{T}_{3}\right)$ & $\left(\mathrm{fT}_{3}\right)$ & \\
& & $\mathrm{nmol} / \mathrm{L}$ & $\mathrm{pmol} / \mathrm{L}$ & $\mathrm{nmol} / \mathrm{L}$ & $\mathrm{pmol} / \mathrm{L}$ & $\%$ \\
\hline
\end{tabular}

Experiment 1: Normal protein level, NP (Protein supply: 100\%)

$\begin{array}{cccccccc}2 \quad \text { CAS+ } & 6 & 37.71 \pm 5.76^{\mathrm{a}} & 13.52 \pm 2.01^{\mathrm{a}} & 1.69 \pm 0.32^{\mathrm{a}} & 0.43 \pm 0.25^{\mathrm{a}} & 4.5 \pm 1.0^{\mathrm{a}} \\ & \text { SPI- } & 6 & 35.58 \pm 6.06^{\mathrm{a}} & 12.51 \pm 2.57^{\mathrm{a}} & 1.63 \pm 0.41^{\mathrm{a}} & 0.72 \pm 0.33^{\mathrm{a}} & 4.6 \pm 1.3^{\mathrm{a}} \\ & & & & & & & \\ & & & & & & & \\ & \text { CAS+ } & 5^{*} & 38.02 \pm 6.93^{\mathrm{a}} & 12.17 \pm 2.40^{\mathrm{a}} & 1.63 \pm 0.30^{\mathrm{a}} & 0.45 \pm 0.30^{\mathrm{a}} & 4.3 \pm 0.8^{\mathrm{a}} \\ & \text { SPI- } & 5^{*} & 38.80 \pm 6.41^{\mathrm{a}} & 14.67 \pm 2.00^{\mathrm{a}} & 1.58 \pm 0.27^{\mathrm{a}} & 0.59 \pm 0.34^{\mathrm{a}} & 4.1 \pm 0.8^{\mathrm{a}}\end{array}$

Experiment 2: Low protein level, LP (Protein supply: $50 \%$ of NP)

$\begin{array}{cccccccc}2 \quad & \text { CAS+ } & 6 & 50.84 \pm 7.93^{\mathrm{a}} & 11.91 \pm 2.23^{\mathrm{a}} & 2.32 \pm 0.40^{\mathrm{a}} & 0.75 \pm 0.33^{\mathrm{a}} & 4.6 \pm 1.2^{\mathrm{a}} \\ & \text { SPI- } & 4^{* \mathrm{*}} & 56.28 \pm 12.20^{\mathrm{a}} & 16.15 \pm 4.10^{\mathrm{b}} & 1.57 \pm 0.31^{\mathrm{b}} & 0.31 \pm 0.18^{\mathrm{b}} & 2.8 \pm 0.7^{\mathrm{b}} \\ & & & & & & & \\ & & & & & & & \\ & \text { CAS+ } & 6 & 43.62 \pm 8.62^{\mathrm{a}} & 9.88 \pm 1.89^{\mathrm{a}} & 2.19 \pm 0.43^{\mathrm{a}} & 1.04 \pm 0.45^{\mathrm{a}} & 5.0 \pm 1.3^{\mathrm{a}} \\ & \text { SPI- } & 4^{* *} & 50.53 \pm 7.00^{\mathrm{a}} & 16.17 \pm 4.10^{\mathrm{b}} & 1.21 \pm 0.21^{\mathrm{b}} & 0.40 \pm 0.25^{\mathrm{b}} & 2.4 \pm 0.6^{\mathrm{b}}\end{array}$

Protcin sources were casein with amino acid supplementation (CAS+) and soy protein isolate without amino acid supplementation (SPI-). Experiments were cross over trials, i. e. after two periods dietary proteins CAS+ and SPI- were replaced by each other. ${ }^{2.6}$ Means with different superscripts within a period and within one column are significantly different $(\mathrm{p}<0.05)$; ' $\mathrm{T}_{4}$, total thyroxine; $\mathrm{T}_{3}$, total triiodothyronine;

$\mathrm{fr}_{3}$, free triiodthyronine, $\mathrm{fT}_{4}$, free thyroxine

- One animal was taken out of experiments due to health problems;

"Two animals were taken out of experiments due to health problems.

ration (30\%) for both protein qualities, and a higher $\mathrm{T}_{3}$ concentration (30\%) for CAS+. Consequently, the $\mathrm{T}_{3} / \mathrm{T}_{4}$ ratio for CAS+ resulted in similar values of approximately $4.4 \%$ as at NP; the ratio for SPI- was decreased to $2.8 \%$ in the $2^{\text {nd }}$ and to $2.4 \%$ in the $4^{\text {th }}$ period.

\section{Discussion}

It is known that thyroid hormones may play a role in mediating the thermogenic response to low protein diets. The decreased energetic efficiency observed in growing rats and pigs fed low protein diets is often associated with a marked rise in $T_{3}$ concentration (TULP et al., 1979, ROTHWELL et al., 1983, GURR et al., 1980), which is frequently interpreted as an adaptive diet-induced thermogenesis. Some energy dissipating systems such as the hepatic mitochondrial $\alpha$-GP shuttle (TYZBIR et al., 1981, SAWAYA and LUNN, 1985, KEAGY et al., 1987), as well as the thermogenic activity of brown adipose tissue (ROTWELL et al., 1983) are sensitive to the thyroid hormone status. Because dietary proteins with a lower biological value induce an increase in $T_{4}$ levels, we propose that similar to protein deficient diets the excess of dietary energy, which can not be deposited as protein, could also be dissipated as heat through increased thermogenesis. 
In the present study at LP the $\mathrm{T}_{4}$ concentrations were slightly but the $\mathrm{fT}_{4}$ concentrations were significantly higher in SPI- than in CAS+ fed pigs within both periods. These findings are in accordance with literature data (CREE and SCHALCH, 1985; FORSYTHE, 1986; BARTH et al., 1988; SCHOLZ-AHRENS et al., 1990; POTTER et al., 1996). At NP, serum concentrations of all thyroid hormones were not affected by the dietary protein quality. The latter finding is surprising, because BARTH et al. (1988) and SCHOLZ-AHRENS et al. (1990) have shown that feeding pigs with diets similar in composition to the NP diets, providing 18, 52 and $30 \%$ of ME from protein, carbohydrate and fat respectively, resulted in significantly higher $\mathrm{T}_{4}$ concentrations in SPI- fed pigs. Reasons for the contrary endocrine response may be due to different pig breeds, age and sex of animals. In the present study growing castrated male pigs of the German Landrace were fed isoenergetic diets, which provided for 2.5 times the maintenance requirement of ME. BARTH et al. (1988) and SCHOLZ-AHRENS et al. (1990) used adult female Göttingen miniature pigs fed isoenergetic diets at the maintenance requirement of ME. However, BARTH et al. (1988) obtained in experiments with growing minipigs fed $15 \%$ soy protein isolate or casein higher values of $\mathrm{T}_{4}$ and $\mathrm{fT}_{4}$ in the SPI- group, but the difference was statistically not significant. These findings correspond better to our findings at the NP level.

The present results indicated that both CAS+ and SPI- fed pigs responded similarly to the low protein feeding regime by exhibiting an increase in total $\mathrm{T}_{4}$ concentration of $30 \%$. Interestingly, the $\mathrm{T}_{3}$ and $\mathrm{fT}_{3}$ levels were only increased in the CAS+ fed pigs. However, we expected results in the opposite direction. But recently, similar inverse relationships between $T_{3}$ concentration and casein diets have been reported by POTTER et al. (1996), who carried out experiments with hamsters. Our findings are partially in disagreement with other literature data (TULP et al. 1979, ROTHWELL et al., 1983), which showed that consumption of low protein diets is associated with an increase in $\mathrm{T}_{3}$ concentration and mostly with no effect on the $\mathrm{T}_{4}$ level. However, interpretation is made difficult, if animals were fed diets to ad libitum intake. In contrast to our findings such data do not allow to conclude unambiguously whether protein supply is responsible for differences in thyroid hormone levels. It is possible that the increased $T_{3}$ level in LP diets was a response to an elevation in food and energy intake (DANFORTH et al., 1979)

Our observations of increased serum $\mathrm{T}_{4}$ concentrations are consistent with findings of ORIEN et al. (1979), who estimated elevations in $\mathrm{T}_{4}$ concentrations in young rats fed protein restricted diets $(8 \%)$ of a normal energy density in comparison to high protein diets $(22 \%)$. Because $\mathrm{T}_{4}$ is synthesised exclusively from the thyroid gland it may be suspected that the observed higher $\mathrm{T}_{4}$ levels may indicate an increase in thyroid secretion induced by dietary protein deficiency. In contrast, ATINMO et al. (1978) observed lower serum $\mathrm{T}_{4}$ concentrations in pigs after feeding low protein diets. Furthermore, the question arises why it comes to contrary endocrine response in $\mathrm{T}_{4}$ concentration after feeding low protein diets. BERGNER (1989) found lower thyroid secretion rates (TSR) in pigs and rats after feeding proteins with a lower biological value. He concluded that the $\mathrm{T}_{4}$ concentration in the blood depends on the thyroid secretion rates and the $T_{4}$ utilization of tissue. From this point of view it can not be 
concluded that higher serum $\mathrm{T}_{4}$ concentrations exclusively derive from a higher TSR.

The ratio $T_{3} / T_{4}$, as a measure of the peripheral deiodination of $T_{4}$, should also be interpreted critically. NOWAK and SLEBODZINSKI (1986) proved that the greatest proportion of the daily $T_{3}$ production derived from peripheral monodeiodination of $T_{4}$ with values ranging between 70 and $80 \%$. In the present study the $T_{4}$ levels at LP were increased in both dietary groups, the CAS+ groups resulted in similar values of $T_{3} / T_{4}$ ratio at both protein levels. Apparently at LP, more $T_{3}$ is derived from $T_{4}$ in the periphery in CAS+ fed pigs, which is also covered by higher $\mathrm{T}_{3}$ values. The $\mathrm{T}_{3}$ levels for SPI- were similar at both protein levels with the consequence of a $50 \%$ reduction in the $\mathrm{T}_{3} / \mathrm{T}_{4}$ ratio at LP. In general, a higher $\mathrm{T}_{4}$ level is followed by a higher $\mathrm{T}_{3}$ level (VOSBERG and WAGNER, 1991). A reason for the contrary endocrine response of SPI- at LP may be due to the lower protein quality of SPI-. As described by LAUTERIO and SCANES (1987), phenylalanine and tyrosine deficiency have been found to decrease circulating $\mathrm{T}_{3}$ concentrations. Since SPI- contains approximately $35 \%$ less of both $\mathrm{AA}$ than $\mathrm{CAS}+$, the lack of an increase in $\mathrm{T}_{3}$ concentration following an increased $\mathrm{T}_{4}$ level in SPI-, in comparison to CAS+, may be explained by the deficiency of these two AA in SPI- at LP.

In summary, our findings indicate that the circulating concentration of thyroid hormones depends primarily on the protein supply and after a decrease of supply to $50 \%$ secondly on the dietary protein quality.

In general, long term feeding of dietary proteins with a lower biological value leads to significant lower growth performance, i.e. protein deposition, than feeding dietary proteins with a higher value (SALTER et al., 1990; ROY et al., 1997; SÉVE et al., 1997). The present results indicate changes in the same manner when feeding SPI- with the lower biological value in comparison to CAS + in all periods at both protein levels. The excess of dietary energy, which cannot be deposited as protein, can either be used for fat deposition (KEAGY et al., 1987) or dissipated as heat (TULP et al., 1979; GURR et al., 1980).

In the present study the total efficiency of utilization of $\mathrm{ME}$ (energy retention/ME intake) within periods at both protein levels was not significantly different between CAS+ and SPI-. Independent on the protein quality and quantity, pigs retained 38 to $42 \%$ of ME. The slightly lower efficiency of utilization of ME (36 vs. $40 \%$ ) in the $1^{\text {st }}$ period of NP, in both feeding groups, was caused by the higher PER in relation to FER (see table 2). However, in general, our findings are in accordance with the data of a review presented by BERGNER and HOFFMANN (1996), who indicated values of 40 to $45 \%$ for growing finishing pigs.

Under the conditions of feeding isoenergetic diets, which provided 2.5 times the maintenance requirement of metabolizable energy (1875 $\mathrm{kJ} \mathrm{ME} /\left(\mathrm{kg} \mathrm{BW}{ }^{0.62} \mathrm{x} \mathrm{d}\right)$ ), the pigs fed SPI- retained less protein energy per unit metabolic BW compared to pigs fed CAS+. However, with one exception, SPI- feeding neither resulted in a significantly higher FER nor in a significantly higher HP. There are several reasons for no observed differences in both of these parameters with regards to CAS+ and SPI-. First there is, on average, a $3 \%$ lower ME intake in SPI fed pigs. Secondly, the changes in PER between SPI- and CAS+ are significant, however, they are expressed as values with an 
average of $46 \mathrm{~kJ} /\left(\mathrm{BW}^{0.62} \mathrm{x} \mathrm{d}\right)$. When taking the value of the standard deviation (on average $53 \mathrm{~kJ} /\left(\mathrm{BW}^{0.62} \mathrm{x} \mathrm{d}\right)$ ) of $\mathrm{HP}$ into consideration, it is evident that the standard deviation in HP is equal or even higher than the difference in PER between CAS+ and SPI-, therefore no significant differences in FER and HP could be expected. In addition the higher excess of ME in SPI-, in comparison to CAS+, seems to be divided into both FER and HP.

Analogous experiments with growing rats fed low protein diets based on SPI- or CAS+ (KLEIN et al., 2000) are not consistent with our findings of a similar energetic efficiency in SPI- and CAS+ fed pigs. The SPI- fed rats retained both less protein energy and fat energy in comparison to CAS-fed rats.

Several experimental results reported in the literature (e.g., MÜLLER and KIRCHGESSNER, 1979; SCHIEMANN et al., 1983; reviewed by KLEIN and HOFFMANN, 1989) demonstrated that the additional energy costs for protein deposition compared to fat deposition are relatively high. The protein deposition is connected to the processes of protein turnover, in which the additional energy costs are mainly caused by higher synthesis rates. MILLWARD et al. (1976) calculated a value of energy costs (ME) for synthesis, which is dissipated as heat, of $0.15 \mathrm{~kJ} / \mathrm{kJ}$ and $3.6 \mathrm{~kJ} / \mathrm{g}$ protein synthezised, respectively. This value is based on the generally accepted energy cost of 5 moles ATP per mole of amino acid incorporated in the peptide chain, and represents the minimum cost of protein synthesis. When considering the relatively high standard deviation in HP, the question arises whether there were differences in the protein synthesis between SPI- and CAS-fed pigs, which produced measurable differences in HP. In the present study measurements of energy balance and protein turnover were carried out simultaneously (SAGGAU et al., 2000). The comparison between CAS+ and SPI- demonstrated that in CAS+ fed pigs, the higher protein deposition was realized by both a higher synthesis and degradation, e.g., enhanced protein turnover. Under consideration of calculated values for minimum energy costs (ME) of protein synthesis (MILLWARD et al., 1976) and the observed differences in protein synthesis between the both dietary groups, no measurable effect on HP can be expected between CAS+ and SPI- within the periods of both dietary levels.

FULLER et al. (1987 a, b) also carried out trials with growing pigs fed daily a constant energy supply with a low in comparison to a high protein level and a variation of protein quality by altering the level of the first limiting AA, lysine, at both protein supply levels. Their data support our conclusion that there was no change in heat production after feeding an AA deficient diet.

As mentioned above, higher $\mathrm{T}_{4}$ and $\mathrm{fT}_{4}$ levels were estimated in SPI- than in CAS+ fed pigs at NP. In addition, both SPI- and CAS+ fed pigs responded similarly to LP by exhibiting an increase in total $\mathrm{T}_{4}$ concentration of $30 \%$. However we could not establish any relationship between increased thyroid hormone concentrations and heat production. HILLGARTNER and ROMSOS (1987) postulated that higher thyroid concentrations resulting from consumption of low protein diets are not directly responsible for activation of adaptive thermogenesis. HILLGARTNER and ROMSOS (1987) carried out experiments with rats fed diets containing 5, 8 or $22 \%$ CAS. The $\mathrm{T}_{3}$ level was increased in the same manner when fed 5 or 8 in comparison to $22 \%$ CAS 
diets but the efficiency of energy retention was reduced in rats fed $5 \%$ CAS, whereas no significant change was observed in rats fed $8 \%$. From these findings, the authors concluded that thyroid hormones play only a permissive role in dissipation of energy in growing rats.

In a recent paper, MOROVAT and DAUNCEY (1998) analysed the thyroid hormone status in growing pigs of the Large White breed after modification of feed intake. Pigs with a total $\mathrm{T}_{4}$ concentration of $37.9 \pm 3.9 \mathrm{nmol} / \mathrm{L}$ were described as euthyroid and those with a concentration of $70 \mathrm{nmol} / \mathrm{L}$ and higher were considered hyperthyroid. From these findings, we concluded that total $\mathrm{T}_{4}$ concentration $(50$ to $56 \mathrm{nmol} / \mathrm{L})$ at $\mathrm{LP}$ were slightly increased but still euthyroid.

Furthermore, the question arises of how an excess of dietary energy can increase either fat deposition or heat production. GURR et al. (1980) demonstrated both metabolic pathways in growing pigs. There were, however, essential differences to our trials. GURR et al. fed either restricted amounts of a high protein diet $(26 \%)$ or to ad libitum amounts of a low protein diet ( $2 \%)$ to pigs of 6 and $20 \mathrm{~kg} \mathrm{BW.} \mathrm{In} \mathrm{all} \mathrm{cases,} \mathrm{pigs} \mathrm{fed} \mathrm{low}$ protein diets consumed approximately three times as much as pigs fed high protein diets. In contrast we fed similar restricted amounts of isoenergetic diets per unit metabolic body weight in all fattening periods of NP and LP which enabled a comparison due to protein intake quantity and/or quality differences alone. GURR et al. (1980) demonstrated that in $20 \mathrm{~kg}$ pigs, almost $70 \%$ of the energy excess caused by feeding the low protein diet was deposited in the carcass as fat, which is in accordance to our findings. In the $6 \mathrm{~kg}$ pigs fed low protein diets, changes in body energy content accounted for only a small fraction $(27 \%)$ of the total energy intake, a large difference in energy expenditure was seen between these animals and the high protein group, which GURR et al. (1980) attributed to differences in dietary-induced thermogenesis. In addition, possible metabolic indicators of diet induced thermogenesis were investigated in the group of $6 \mathrm{~kg}$ pigs. At the low protein supply these pigs showed higher plasma $T_{3}$ levels and hepatic mitochondrial $\alpha$-glycerophosphate dehydrogenase activity. It must be taken into account that at LP the ratio of protein energy to total energy intake was drastically reduced and corresponded to a supply as in Kwashiorkor patients (COWARD and LUNN, 1981; SCHOPPE, 1988). In these metabolic situation very young pigs seemed to dissipate the excess of energy mainly as heat through increased thermogenesis. It is apparent from the study of DANFORTH et al (1979) on humans that the intake of high caloric amounts, e.g. above the energetic requirement, also increases $\mathrm{T}_{3}$ concentrations, which may be associated with an increased thermogenesis.

ROTHWELL et al. (1983) speculated that the thermogenic activity of brown adipose tissue (BAT) may play an important role in diet-induced thermogenesis when low protein diets are fed to rats. The BAT contains a tissue-specific mitochondrial uncoupling protein (UCP), which is sensitive to thyroid hormones. KEAGY et al. (1987) postulated that protein-deficient chickens cannot dispose a surplus of dietary energy by this mechanism, because most birds apparently lack BAT. It is known, that pigs also do not contain BAT (TRAYHURN et al., 1989) and therefore they cannot use this mechanism. However, recently in white adipose tissue a similar tissue-specific mitochondrial uncoupling protein (UCP 2) was discovered (FLEURY et al., 1997). 
It is concluded that, generally, HP was neither clearly affected by the protein quality nor by the quantity and resulted almost in identical values of $60 \%$ of $\mathrm{ME}$ intake. The increased thyroid hormone levels at LP level were still in euthyroid range of pigs and obviously not associated with HP.

\section{References}

ATINMO, T.; BALDIJAO, C.; POND, W. G.; BARNES, H.:

The effect of dietary protein restriction on serum thyroxine levels of pregnant or growing swine. J. Nutr. 108 (1978), 1547-1553

BARTH, C. A.; SCHOLZ-AHRENS, K. E.; PFEUFFER, M.; DE VRESE, M.: Endocrine response to animal and vegetable protein. In: C.A. BARTH and E. SCHLIMME (Eds.) Milk BERGNER, H.: protein: nutritional, functional and technological aspects. Springer-Verlag, New York, (1988), 62-67

N-Stoffwechsel und seine Regelmechanismen. Arch. Anim. Nutr. 39 (1989), 377-392

BERGNER, H.; HOFFMANN, L.:

Bioenergetik und Stoffproduktion landwirtschaftlicher Nutztiere. Harwood Academic Publisher, Chur, Schweiz (1996), 327-329

BROUWER, E.:

Report of sub-committee on constants and factors. In: K. L. BLAXTER (Ed.): Energy metabolism. Proc $3^{\text {rd }}$ Symp. on Energy Metabolism, Troon, U.K., Academic Press London, EAAP - Publ. No. 11 (1965), 441-443

COWARD, W.A.; LUNN, P.G.:

The biochemistry and physiology of Kwashiorkor and Marasmus. Br. Med. Bull. 37 (1981), 19-24

CREE, T.C.; SCHALCH, D.S.:

Protein utilization in growth: Effect of lysine deficiency on serum growth hormone, somatomedins, insulin, total thyroxine $\left(\mathrm{T}_{4}\right)$ and triiodothyronine $\left(\mathrm{T}_{3}\right)$, free $\mathrm{T}_{4}$ index, and total corticosterone. Endocrinology, 117 (1985), 667-673

DANFORTH, E.JR.; HORTON, E.S.; O'CONNELL, M.:

Dietary-induced alterations in thyroid hormone metabolism during overnutrition. J. Clin. Invest. 64 (1975), 1336-1347.

FLEURY, C.; NEVEROVA, M.; COLLINS, S.; RAIMBAULT, S.; CHAMPIGNY, O.; LEVI-MEYRUEIS, C.; BOUILLAUD, F.; SELDIN. M.F.; SURWIT, R.S.; RUCQUIER, D.; WARDEN, CH.: Uncoupling protein-2: A novel gene linked to obesity and hyperinsulinemia. Nature Genetics 15 (1997), 269-272

FORSYTHE, W.A.:

Comparison of dietary casein or soy protein effects on plasma lipids and hormone concentrations in the gerbil. J. Nutr. 116 (1986), 1165-1167

FULLER, M.F.; CADENHEAD, A.; MOLLISON, G.; SÉVE, B.:

Effects of the amount and quality of dietary protein on nitrogen metabolism and heat production in growing pigs. Br. J. Nutr. 58 (1987a), 277-285

FULLER, M.F.; REEDS, P.J.; CADENHEAD, A.; SÉVE, B.:

Effects of the amount and quality of dietary protein on nitrogen metabolism and protein turnover of pigs. Br. J. Nutr. 58 (1987b), 287-300

GESELLSCHAFT FÜR ERNÄHRUNGSPHYSIOLOGIE DER HAUSTIERE (GfEH): Energie- und Năhrstoffbedarf landwirtschaftlicher Nutztiere Nr. 4: Versorgungsempfehlungen für Mastschweine. DLG-Verlag, Frankfurt, Main (1987)

GURR, M.I.; MAWSON, R.; ROTHWELL, N.J.; STOCK, M.J.:

Effects of manipulating dietary protein and energy intake on energy balance and thermogenesis in pigs. J. Nutr. 110 (1980), 532-542

HILLGARTNER, F. B.; ROMSOS, D.R.:

Iodothyronine 54- deiodination in rats fed low protein diets: Lack of correlation with energy balance. J. Nutr. 117 (1987), 368-375

HOFFMANN, L.; SCHIEMANN, R.:

Von der Kalorie zum Joule: Neue Großenbeziehungen bei Messungen des Energieumsatzes und bei der Berechnung von Kennzahlen der energetischen Futterbewertung. Arch. Anim. Nutr. 30 (1980), 733-742 
HOFFMANN, L.; BEYER, M.; JENTSCH, W.:

Abhängigkeit von Brutto-, Verdaulicher und Umsetzbarer Energie des Futters sowie des Energieansatzes vom Gehalt an verdaulichen Năhrstoffen unter Berllcksichtigung von Stărke und Zucker. Ein Beitrag zur Weiterentwicklung der energetischen Futterbewertung. Ergebnisse aus Untersuchungen mit Schweinen. Arch. Anim. Nutr. 44 (1993), 123-137

KEAGY, E.M.; CAREW, F.; ALSTER F.A.; TYZBIR, R.S.:

Thyroid function, energy balance, body composition and organ growth in protein-deficient chicks. J. Nutr. 117 (1987), 1532-1540

KLEIN, M.; HOFFMANN, L.:

Bioenergetics of protein retention. In: BOCK, H.-D.; EGGUM, B.O.; LOW, A.G.; ZEBROWSKA, T. (Eds.): Protein Metabolism in Farm Animals. Oxford University Press, VEB Deutscher Landwirtschaftsverlag, Berlin (1989), 404-440

KLEIN, M.; SCHADEREIT, R.; KÜCHENMEISTER, U.:

Energy metabolism and thyroid hormone levels of growing rats in response to different dietary proteins - soy protein or casein. Arch. Anim. Nutr., 53 (2000), 99-125

LAUTERIO, T.J.; SCANES, C.G.: Hormonal responses to protein restriction in two strains of chickens with different growth characteristics. J. Nutr. 117 (1987), 758-763

MILLWARD, D.J.; GARLICK, P.J.; REEDS, P.J.:

The energy cost of growth. Proc. Nutr. Soc. 35 (1976), 339-349

MÜLLER, H.L.; KIRCHGESSNER:

Zur Energetik der Proteinsynthese beim Wachstum. Z. Tierphysiol., Tierernăhrg., Futtermittelkde. 42 (1979), 161-167

MOROVAT, A.; DAUNCEY, M.J.:

Effects of thyroid status on insulin-like growth factor-I, growth hormone and insulin are modified by food intake. Eur. J. Endocrinol. 138 (1998), 95-103

NOWAK, G., SLEBODZINSKI, A. B.,: Extrathyroidal conversion of thyroxine to 3,5,3' - triiodothyronine $\left(\mathrm{T}_{3}\right)$ and 3,3',5' - triiodothyronine $\left(\mathrm{rT}_{3}\right)$ and its contribution to total triiodothyronines production rates in fed and food restricted piglets. J. Vet. Med. A, 33 (1986), 337-348

ORIEN, L.; TULP, P.; KRUPP, E.; DANFORTH, E. JR.; HORTON, E.S.: Characteristics of thyroid function in experimental protein malnutrition. J. Nutr. 109 (1979), 1321-1332

POTTER, S.M.; PERTILE, J.; BERBER-JIMENEZ, M.D.: Soy protein concentrate and isolated soy protein similarly lower blood senum cholesterol but differently affect thyroid hormones in hamsters. J. Nutr. 126, (1996), 2007-2011

ROTHWELL, N.J.; STOCK, M.J.; TYZBIR, R.S.: Mechanism of thermogenesis induced by low protein diets. Metabolism 32 (1983), 257-261

ROY, N.; LAPIERRE, $\mathrm{H}$. and BERNIER, J.F.: Effect of lysine deficiency on whole-body protein metabolism in growing pigs. Proc. Nutr. Soc. 56 (1997), 176

SAGGAU, E.; SCHADEREIT, R.; BEYER, M.; SUSENBETH, A.; VOIGT, J.; HAGEMEISTER, H.: Effects of casein and soy protein isolate on protein turnover in growing pigs fed on a constant energy supply. $15^{\text {th }}$ Symposium on Energy Metabolism in Animals in Elsinore, Denmark (2000), accepted

SALTER, D.N.; MONTGOMERY, A.I.; HUDSON. A.; QUELCH, D.B.; ELLIOTT, R.J.: Lysine requirements and whole-body turnover in growing pigs. Br. J. Nutr. 63 (1990), 503-513

SAWAYA A.L.; LUNN P.G.: Evidence suggesting that elevated plasma triiodothyronine concentration of rats fed on protein deficiency diets is physiologically active. Br. J. Nutr. 53 (1985), 175-181

SCHIEMANN, R.; BOCK, H.D.; KELLER, J.; HOFFMANN, L.; KRAWIELITZKI, K.; KLEIN, M.: Methodische Untersuchungen zum Eiweißumsatz und zur Bioenergetik des Eiweißansatzes bei wachsenden Tieren. 1. Mitteilung: Bestimmung von Parametern des Wachstums und des Eiweißansatzes von Kuken nach Langzeitmarkierung mit ${ }^{15} \mathrm{NH}_{4}$-Azetat. Arch. Anim. Nutr. 33 (1983), $57-74$

SCHOLZ-AHRENS, K.E.; HAGEMEISTER, H.; UNSHELM, J.; AGERGAARD, N.; BARTH, C.A.: Response of hormones modulating plasma cholesterol to dietary casein or soy protein in minipigs. J. Nutr. 120 (1990), 1387-1392 
SCHOPPE, 1.:

Die Reaktion des N-Haushaltes und der Schilddrusenhormone auf Casein- und Sojadiäten. Univ. Kiel, Diss., 1988

SÉVE, B.; PONTER, A.A.:

Nutrient-hormone signals regulating muscle protein turnover in pigs. Proc. Nutr. Soc. 56 (1997), 565580

Statistical Package for the Social Science (SPSS):

Version 7.5 for windows, Chicago, Illinois (1997)

TRAYHURN, P.; TEMPLE, N.J.; VAN AERDE, J.:

Is brown adipose tissue present in the pig? Proc. Nutr. Soc. 49 (1990), $132 \mathrm{~A}$

TULP, O.L.; KRUPP, P.P.; DANFORTH, E. JR.; HORTON, E.S.:

Characteristics of thyroid function in experimental protein malnutrition. J. Nutr. 109 (1979), 1321-1332

TYZBIR, R.S.; KUNIN, A.S.; SIMS, N.M.; DANFORTH, E. JR.:

Influence of diet composition on serum triiodothyronine $\left(\mathrm{T}_{3}\right)$ concentration, hepatic mitochondrial metabolism and shuttle system activity in rats. J. Nutr. 111 (1981), 252-259

VERBAND DEUTSCHER LANDWIRTSCHAFTLICHER UNTERSUCHUNGS- UND FORSCHUNGSANSTALTEN (VDLUFA):

Methodenbuch Band 3, VDLUFA-Verlag, Darmstadt (1988)

VOSBERG, H.; WAGNER, H.:

Schilddrilsenkrankheiten: Diagnostik und Therapie. Georg Thieme Verlag Stuttgart - New York, (1991), 16-18

Received: $2000-08-30$

Accepted: $2000-09-29$

Authors' address

Dr. ELKE SAGGAU, Dr. habil. MANFRED BAYER, Dr. MONIKA KLEIN,

Dr. RUTHILD SCHADEREIT, Dr. MICHAEL DERNO, Dr. habil. WERNER JENTSCH,

Dipl. math. HELMUT SCHOLZE

Forschungsinstitut fur die Biologie landwirtschaftlicher Nutztiere (FBN)

Wilhelm-Stahl-Allee 2

D-18196 Dummerstorf

Germany 
Arch. Tierz., Dummerstorf 43 (2000) 6, 648

\title{
Buchbesprechung
}

\section{Pferderassen der Welt}

\author{
WOLFGANG KRESSE
}

368 Seiten, 280 Farbfotos, 12 Zeichnungen, Verlag Eugen Ulmer, Stuttgart, 1999, ISBN 3-8001-7392-1, 49,80 $\mathrm{DM}, 364,00 \mathrm{dS}, 46,00 \mathrm{sFr}$

In einer Zeit zunehmender Technisierung und elektronischer Medien in allen Arbeits- und Lebensbereichen erhalt dic Bezichung vicler Menschen zu Haustieren einen immer großeren Stellenwert. Das gilt in besonderer Weise fur das Pferd. Durch das vorliegende Buch ist der Verlag einmal mehr diesem Bedurfnis in gelungener Weise nachgekommen. Dieses Buch zeichnet ein Gesamtbild der faszinierenden Vielfalt vorhandener Pferderassen. In der gelungenen Kombination von Wort und Bild vermittelt es Wissenswertes uber mehr als 300 Rassen. Es ist nicht einfach die Pferde- und Ponyrassen, so sie denn uber ein Stutbuch verfugen und Pferdetypen, die nach ihrem Nutzungszweck definiert werden, zu klassifizieren. Das war auch nicht das Ziel des Autors, dem es darum ging dem Leser in gebotener Kürze einen umfassenden Überblick über ca. 320 Pferde- und Ponyrassen der Welt zu geben und sie in diesem Bilderatlas in alphabetischer Reihenfolge aufzufuhren. Dass es ihm dabei gelang dieses Weltrassenspektrum, neben den wichtigsten Angaben zu Exterieur, Verbreitung, Leistungsmerkmalen, Verwendung und Zuchtgeschichte, meist auch durch farbige Abbildungen vorzustellen, ist ein besonderes Verdienst. Es macht dieses Buch daher auch für Leser interessant, die Freude an schönen Tierbildern haben und nicht direkt mit der Pferdehaltung liiert sind.

In den ersten einfuhrenden, allgemeinen Buchabschnitten werden Ursprung des Pferdes, das Pferd in vorgeschichtlicher Zeit, das frühgeschichtliche Pferd und die Entwicklung der Rassen vorgestellt. Informationen über „Das Pferd macht Weltgeschichte" und „Das Pferd in der Kulturgeschichte" beschließen diesen mit einer Fülle von Informationen zusammengestellten Buchteil. Sie belegen, dass im Vergleich zu den meisten anderen Haustieren, das relativ spăt domestizierte Pferd die Menschen bis in die Gegenwart in unterschiedlicher Weise auf allen ihren Wegen begleitet hat und ihnen ein zuverlässiger Partner geworden ist. Im Hauptteil des Buches werden die Rassen vorgestellt. Der Leser findet für jede Rasse in komplexer, ubersichtlicher und fundierter Weise, sich auf das Wesentliche beschränkende Informationen. Die Textaussagen werden durch ausdrucksvolle Farbfotos unterstützt. Ergänzt wird dieser farbige Bildatlas durch ein umfangreiches internationales Adressenverzeichnis sowie eine Literaturubersicht.

Dieses Buch hält nicht nur fur Pferdezllchter, -freunde und -liebhaber eine Fulle von sorgfaltig zusammengetragenen Informationen bereit. Es verschafft einen umfassenden Überblick über die bemerkenswerte Vielfalt des Weltrassenspektrums. Als unverzichtbares Nachschlagewerk fur jeden ambitionierten Pferdefreund ist es uneingeschrłnkt Tierliebhabern und vielen weiteren interessierten Lesern zu empfehlen.

ERNST RITTER, Dummerstorf 